
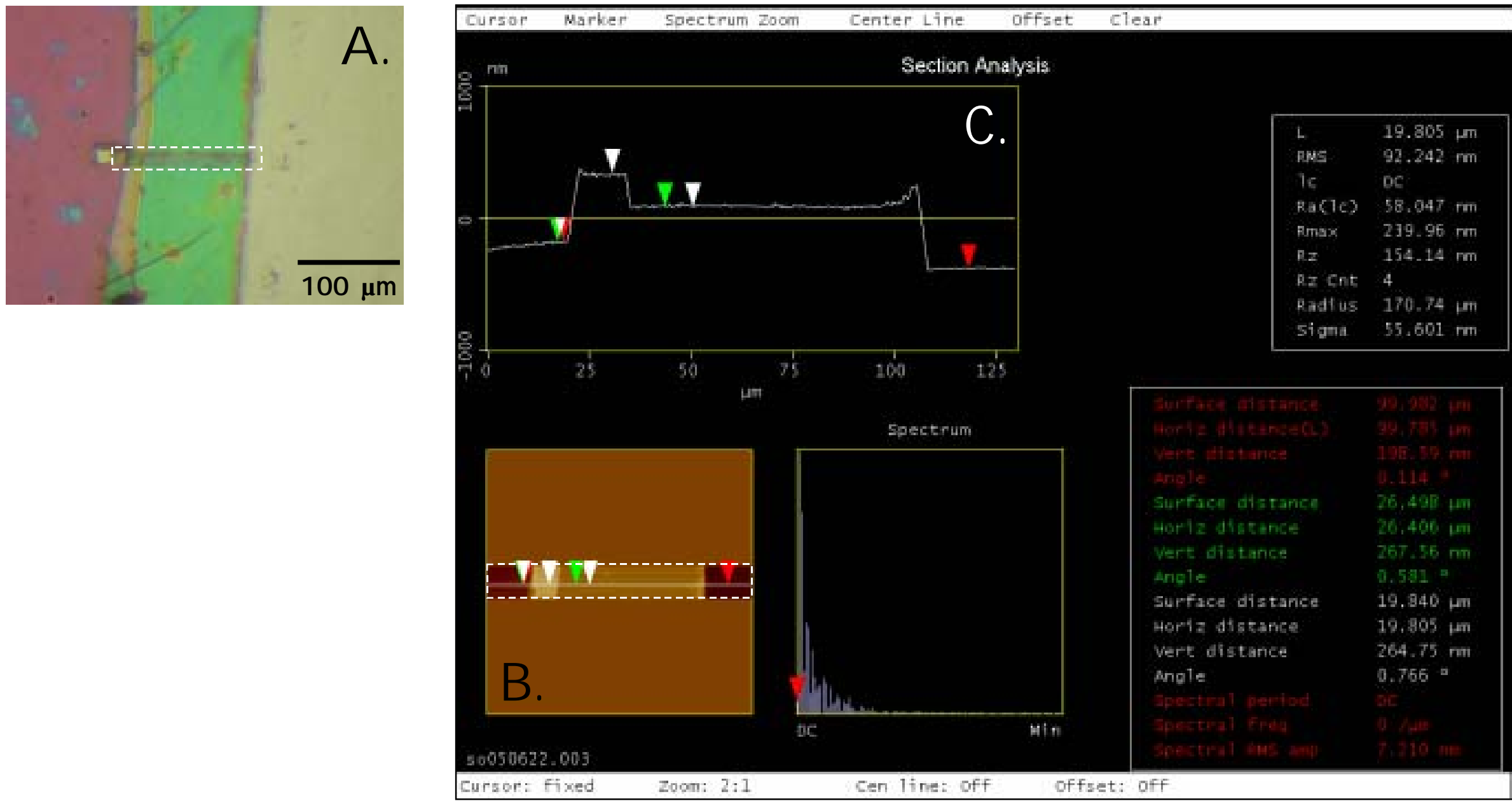

\title{
Supporting Data:
}

Measurement of the thickness of the self-standing membrane released from the hybrid film whose architecture is $\mathrm{SiO}_{2} / \mathrm{PEI} /(\mathrm{PAA} / \mathrm{PEG})_{9} \mathrm{PAA}$ (PAH/PSS) 80 with AFM. A) Optical microscope image of the edge of the membrane refixed on the substrate. B) Top-view tapping-mode AFM image C) Cross-sectional profile along the line in B). 